\title{
Stress control training for older women with metabolic syndrome
}

\begin{abstract}
The aim of this study was to ascertain if Lipp's Stress Control Training would promote beneficial alterations in risk factors associated with metabolic syndrome, which is characterized by the presence of several risk factors, such as: glucose intolerance, dyslipidemia and hypertension, inadequate diet, sedentariness, tobacco use, alcoholism and excessive stress. The study was conducted on 45 elderly women with MetS, whose ages range from 60 to 75 years. They were subjected to Lipp's Stress Control Training, which consisted of 8 two-hourly sessions with 5 groups of 8 participants, once a week. After the intervention, a significant reduction in stress was found $(\mathrm{p}=0.005)$. There was also a significant reduction in before and after total cholesterol $(p=0.012)$, LDL $(p=0.0133)$ and triglycerides $(p=0.05)$ in the group. Stress Control Training reduced some of the MetS risk factors, such as stress, and may have contributed to the reduction in MetS components such as glucose, triglycerides, total cholesterol and LDL cholesterol. If the present data are confirmed in future studies, performed on a larger number of participants, Stress Control Training will be a beneficial, psychological treatment model representing a prophylactic measure for coronary diseases, inasmuch as it could reduce the risk factors involved in MetS.
\end{abstract}

Volume 4 Issue 2 - 2019

\author{
Marilda EN Lipp \\ Instituto de Psicologia e Controle do Stress IPCS, São Paulo, \\ Brazil
}

Correspondence: Marilda EN Lipp, Instituto de Psicologia e Controle do Stress - IPCS, São Paulo, Brazil, Tel I999830535I, Emailmlipp@estresse.com.br

Received: March 01, 2019 | Published: April 05, 2019

Keywords: metabolic syndrome, risk factors, stress

\section{Introduction}

Metabolic syndrome (MetS) is a disorder associated with a significant increase in cardiovascular mortality. ${ }^{1,2}$ MetS affects up to $25 \%$ of the population in the United States and continues to spread, becoming a major clinical and public health problem. ${ }^{3}$

\section{Metabolic syndrome}

This is characterized by the concurrent presence in an individual of at least three of the following components: abdominal obesity, glucose intolerance/insulin resistance, dyslipidemia and hypertension. Inadequate diet, sedentariness, tobacco use, alcoholism and excessive stress are all risk factors in the development of MetS. ${ }^{4}$

\section{Stress as a risk factor in MetS}

Stress is defined as the organism's physical and emotional response to situations that demand a great effort for adaptation. Hans Selye $e^{5}$ proposed that the stress process occurs in three phases: alarm (alert), resistance and exhaustion, each of which is related to specific reactions. Later studies, conducted in Brazil by Lipp, led to the establishment of a new stress phase between the resistance and exhaustion stages, which was called the near-exhaustion phase. ${ }^{6}$ The near-exhaustion phase comes at the end of the resistance phase cited by Selye and provides an opportunity for the development of pathologies which precede exhaustion. Each stress phase embodies specific symptoms, and progression through the phases indicates a worsening picture, eventually leading to the development of diseases to which the individual is predisposed.

Stress has been found to be particularly present in MetS., ${ }^{7.8}$ Studies show that higher levels of stress impact the prevalence of metabolic syndrome (MetS) and coronary heart disease. ${ }^{3}$ Lambert et al. ${ }^{9}$ investigated the relationship between sympathetic activation and psychological stress in patients with MetS and high blood pressure, concluding that mental stress regulates sympathetic activity and could represent a high cardiovascular risk for these individuals. Although epidemiological data suggest strong associations between chronic stress exposure and metabolic disease, most studies only focus on the physical aspects of the syndrome. Interventions which seek to improve quality of life by means of reducing stress are seen as a necessary part of the prevention and treatment of MetS. The aim of this study was to ascertain if Lipp's Stress Control Training (LSCT) would promote beneficial alterations in the risk factors with a group of older women.

\section{Lipp's stress control training (LSCT)}

The proposition of the Stress Control Training is one of lifestyle change and, as such, it could be useful in the treatment of MetS. Created by Lipp, ${ }^{10}$ it has been used in numerous studies and has proved to be effective in the control of stress and the treatment of pathologies such as psoriasis, ulcerative rectocolitis and hypertension. ${ }^{11}$ It consists of 8 weekly sessions each lasting 90 minutes. Professionals from the spheres of nutrition, medicine and physical education may be invited to participate in some of the sessions, since guidance on diet and physical exercise is part of the Stress Control Training. It is a cognitivebehavioral treatment that seeks to reduce the effects of stress, which involves creating patient awareness of their external and internal sources of stress and the learning of diaphragmatic deep breathing techniques, progressive relaxation and cognitive-behavioral strategies to control stress. As part of the training, patients receive strategies on coping and how to reduce sources of stress. It emphasizes change in life style and in the way of perceiving life's struggles.

\section{Objective}

The overall objective of this study was to ascertain if Lipp's Stress Control Training (LSCT) would reduce stress and other biological risk factors in older women. 
The risk factors investigated were: presence of stress (stress in the alarm, resistance, near-exhaustion and exhaustion phases were taken into consideration), levels of cholesterol, triglycerides, blood pressure and glucose, by comparing before and after measurements. The hypothesis that permeated the study was that, after Stress Control Training, there should be a reduction in the base risk factors for the components of MetS.

\section{Method}

\section{Participants}

The study was conducted on 45 women attending a day clinic at a health center in the city of São Paulo, in Brazil. They had all been diagnosed with MetS and were referred by two endocrinologists. The mean age was 61.5 years, with ages ranging from 60 to 75 years.

\section{Instruments}

\section{Lipp's stress symptom inventory for adults- LSSI (Lipp6)}

Instrument created based on the author's experience in the area of stress, clinical practice and research, with the aim of accomplishing the following objectives: to identify the presence, detectable via the inventory, of increased levels of stress; and to identify the stress phase in which the individual is situated: alert, resistance, near-exhaustion or exhaustion.

\section{Psychological interview}

The Interview sought to trace the sociodemographic profile of the participants and investigate the presence of risk behavior for the development or aggravation of the MetS components, such as tobacco use and the excessive consumption of alcoholic beverages.

Laboratory material for the total cholesterol, fractions, triglycerides and glucose examinations and Sphygmomanometer to check Blood Pressure.

\section{Procedure}

After individual psychological assessment, including the application of the LSSI, participants were divided into 5 groups of 8 or 9 . The SCT was conducted in these groups over a period of 8 weeks. Each session lasted 2 hours. Laboratory examinations (levels of total cholesterol and fractions, triglycerides and fasting glycemia) were taken the day before the first session and at the completion the study. As Lipp's Stress Control Training is a multidisciplinary study, it involved one session each with the presence of an endocrinologist, a nutritionist and a physical education instructor. The information and suggestions provided by the respective professionals were reinforced in all subsequent sessions in order to ensure adherence to these practices. The medical treatment was maintained as usual.

\section{Results}

It was found that, at the beginning of the study, the prevalence of stress was $58 \%$ in the resistance phase and $42 \%$ in the near-exhaustion phase. Comparative statistical analysis of stress evaluation before and after TCS demonstrated that there was a significant reduction in the stress level $(\mathrm{p}<0.0001)$ with $15 \%$ of the participants in the resistance phase and $10 \%$ in the near-exhaustion phase.
As far as the analysis of the biological indicators is concerned, the following data were noted:

1. Glucose: At the end of the SCT, $37.5 \%$ of the sample presented a reduction in glucose levels, and $54.16 \%$ fell within the normal range $(70-110 \mathrm{mg} / \mathrm{dl})$.

2. Total Cholesterol: $70.8 \%$ of the participants showed a reduction and $75 \%$ came within the acceptable range $(<200 \mathrm{mg} / \mathrm{dl})$

3. HDL-c: $20.83 \%$ of the sample showed a reduction and $25 \%$ was within the normal range

4. LDL-c: $58.33 \%$ of the women showed a reduction and $87.5 \%$ came within the normal range $(>130 \mathrm{mg} / \mathrm{dl})$

5. Triglycerides: $75 \%$ of the sample showed a reduction and $79.1 \%$ was within the normal level $(160 \mathrm{mg} / \mathrm{dl})$.

Hypertension: the data did not indicate any significant changes in this area.

\section{Conclusion}

The present study produced results which confirmed the hypothesis proposed. After Lipp's Stress Control Training, there was a reduction in some risk factors involved in MetS. These results showed that stress control techniques, including changes in life style, could be used as a complementary treatment in cases of older women suffering from metabolic syndrome.

\section{Acknowledgments}

None.

\section{Conflicts of interest}

Author declares there is no conflict of interest in publishing the article.

\section{References}

1. Gami AS, Witt BJ, Howard DE, et al. Metabolic syndrome and risk of incident cardiovascular events and death. A systematic review and metaanalysis of longitudinal studies. J Am Coll Cardiol. 2007;49:403-414.

2. International diabetes federation (IDF). The IDF consensus worldwide definition of the metabolic syndrome. 2006.

3. Janczura M, Grazyna B, R Nowobilski, et al. Syndrome with stress, coronary heart disease and pulmonary function - An occupational cohortbased study. PLoS One. 2015;10(8):e0133750.

4. Bhatti GK, Bhadada SK, Vijayvergiya R, et al. metabolic syndrome and risk of major coronary events among the urban diabetic patients: north indian diabetes and cardiovascular disease study-NIDCVD-2. J Diabetes Complications. 2016;30(1):72-78.

5. Selye H. The stress of life. McGraw, Hill Education. 1956.

6. Lipp MEN. Inventário de sintomas de stress de lipp. SP: Casa do psicólogo. 2000 .

7. Hjemdahl P. Stress and the metabolic syndrome an interesting but enigmatic association. Circulation. 2002;106:2634-2636.

8. Tamashiro KL, Sakai RR, Shively CA, et al. Chronic stress, metabolism, and metabolic syndrome. Stress. 2011;14(5):468-474.

9. Lambert E, Dawood T, Straznicky N, et al. Association between the 
sympathetic firing pattern and anxiety level in patients with the metabolic syndrome and elevated blood pressure. Journal of Hypertension. 2010;28(3):543-550

10. Lipp MEN. O Valor do controle do stress como terapêutica da hipertensão arterial In: M Nobel, edition. psicossomática campinas: NEP;1991:86-163.
11. Malagris LEN, Brunini TMC, Moss MB, et al. Evidências biológicas do treino de controle do stress em pacientes com HA. Psicologia: Reflexão e Crítica. 2009;22(1):1-9. 